\title{
Desenvolvimento pós-embrionário de Chrysomya putoria (Calliphoridae) em carne equina e sua importância para a entomologia e veterinária forenses
}

[Post embryonic development of Chrysomya putoria (Calliphoridae) using horse meat and its importance for forensic entomology and veterinary ]

V.M. Carraro ${ }^{1}$, I.L.S. Cruz $^{2}$, A. Ururahy-Rodrigues ${ }^{1}$, M. Maleck ${ }^{1,2^{*}}$

${ }^{1}$ Universidade de Vassouras - Vassouras, RJ

${ }^{2}$ Instituto Oswaldo Cruz, Fiocruz - Rio de Janeiro, RJ

\section{RESUMO}

Devido à importância médica, veterinária, ambiental e forense do califorídeo Chrysomya putoria (Diptera), foi avaliado o desempenho apresentado por estágios imaturos criados em carne de cavalo. Os espécimes foram colocados em câmaras aclimatizadas reguladas a $30{ }^{\circ} \mathrm{C}, 60 \pm 10 \%$ UR e 14 horas de fotofase. Os estágios larval e pupal concluíram seu desenvolvimento em 4,16 e 4,12 dias, respectivamente. As larvas pós-alimentação e pupas com $24 \mathrm{~h}$ de idade pesaram, em média, 58,06 e 40,10mg, respectivamente. As taxas de emergência registradas foram de $65 \%$ nas condições experimentais propostas. Como os compostos orgânicos voláteis são diferentes na decomposição de um animal para outro, este trabalho e outros realizados com a mesma dieta são os mais apropriados para estimativa de intervalo post mortem envolvendo cavalos.

Palavras-chave: dieta alternativa, Diptera, mosca varejeira

\begin{abstract}
Due to medical, veterinary, environmental and forensic importance of the calliphorid Chrysomya putoria (Diptera), the performance presented by immature stages, reared on horse meat, was evaluated. The specimens were placed in acclimatized chambers regulated at $30{ }^{\circ} \mathrm{C}, 60 \pm 10 \% \mathrm{RU}$ and 14 hours of photo phase. The larval and pupal stages completed their development in 4, 16 and 4, 12 days, respectively. Postfeeding larvae and aged $24 \mathrm{~h}$ pupae weight on average 58, 06 and 40, $10 \mathrm{mg}$, respectively. The emergence rates recorded were $65 \%$ under the experimental conditions proposed. As volatile organic compounds are different in the decomposition of one animal to another, this work and others carried out with the same diet are the most appropriate for estimating post-mortem interval involving horses.
\end{abstract}

Keywords: alternative diet, Diptera, blowfly

\section{INTRODUÇÃO}

Chrysomya putoria (Wiedemann, 1830), califorídeo exótico, apresenta considerável importância ecológica, médico-veterinária, sanitária e forense. Essa relevância multidisciplinar se deve ao papel da espécie como decompositora de matéria orgânica, vetora mecânica de patógenos e produtora primária e secundária de miíases nos homens e nos animais, e, finalmente, na medicina legal, como ferramenta pericial, por contribuir com a estimativa de intervalo pós-morte, determinação do modo da morte, detecção de negligência e maus-tratos e como prova criminal (Imbiriba et al., 1977; Guimarães e Papavero, 1999; Baltazar et al., 2011; Oliveira Costa, 2013).

Lockwood et al. (1994) estudaram um caso em que a entomologia forense forneceu importantes

Recebido em 11 de outubro de 2019

Aceito em 10 de abril de 2020

*Autor para correspondência (corresponding author)

E-mail: marise.maleck@gmail.com 
informações que ajudaram a esclarecer um episódio relacionado a um cavalo morto há mais de 400 anos. Durante a construção de uma autoestrada a sudeste de Wyoming, foi encontrada uma ossada de cavalo Equus cabalus L., e, após os primeiros exames antropológicos, suspeitou-se de que a morte não tivesse sido natural. Esse fato poderia indicar sinais da realização de um ritual indígena, pois foram encontrados animais desconhecidos posicionados junto à ossada. A sucessão entomológica dos insetos encontrados apontou a estação do ano na qual a morte teria ocorrido e a análise por $\mathrm{C} 14$ situou a morte no século XVII.

McGarry et al. (2017) promoveram o treinamento de veterinários para a aplicação de entomologia forense no bem-estar de animais domésticos. Esse estudo apresentou três relatos de casos, que utilizaram carcaças de coelho, gato e cachorro, dos quais o IPM foi calculado com base nos grausdia acumulados, requeridos para $C$. putoria. $\mathrm{Na}$ decomposição de diferentes modelos de carcaças animais, comparadas com a de humanos, encontram-se diferenças qualitativas nos compostos orgânicos voláteis liberados, que não são iguais aos modelos usados normalmente nos estudos de antropologia forense e tafonomia. Esses resultados apontam para um cuidado maior na escolha de dietas para estudos de biologia de insetos de importância forense (Cablk et al., 2012).

Seu envolvimento em processos de miíases nos animais e no homem foi revisto por Zumpt (1965). Técnicas para a esterilização dos ovos de $C$. putoria foram testadas para que se pudesse usar suas larvas em terapia larval (Ferraz et al., 2012). Importantes estudos estão sendo realizados acerca do papel de suas larvas em investigações criminais (Thyssen e Grella, 2011). Furlanetto et al. (1984) coletaram essa espécie em bancas de pescado, em São Paulo, e isolaram, a partir de dissecações, Salmonella agona.

Outros estudos demonstram o potencial de Chrysomyinae na veiculação de poliovírus dos tipos I, II e III, Entamoeba histolytica, Coxsackie vírus, Shigella sp. e ovos de vários cestódeos (Hall, 1948; Williams, 1954; Greenberg, 1971, 1973), e corroboram a urgência da popularização da educação sanitária solicitada por Cunha e Lomônaco (1996). Estes pesquisadores monitoraram o impacto ambiental provocado pela dispersão de moscas, em Uberlândia, MG: $C$. putoria e Musca domestica L. (Diptera, Muscidae) foram as espécies mais frequientes e abundantes. Em Goiânia, esse califorídeo exótico, que apresentou expressiva capacidade de dispersão pelo território nacional, durante a década de 1980 (Ferreira, 1978; Carvalho e Couri, 1991), encontrava-se fortemente associado à antropobiocenose (Ferreira, 1983), sendo coletado, inclusive, no interior de residências. Prado e Guimarães (1982) distinguiram a alta prolificidade e a falta de especialização alimentar durante o estágio larval como características que provavelmente favoreceram a grande capacidade inicial de dispersão apresentada por $C$. putoria. Entretanto, os índices de sinantropia estimados para essa espécie, no país, motivam controvérsias entre os especialistas (Carraro, 1995).

Fezes e cadáveres, utilizados como fonte alimentar, caracterizam a dicotomia básica apresentada pelos muscoides decompositores, durante a seleção de alimentos: relativamente poucos insetos alimentam-se em ambos, enfatizou Hanski (1987), em sua criteriosa revisão. A incidência significativa de $C$. putoria em aviários é periodicamente destacada. Lá, essa espécie alimenta-se de carcaças de aves, esterco acumulado e ovos quebrados (Cunha e Lomônaco, 1996; Hulley, 1983; Sereno e Neves, 1993; Lomônaco e Prado, 1994). Contrariando os resultados obtidos por Madeira et al. (1982) e D'Almeida e Fraga (2007), a sequência de coletas de adultos dessa espécie, efetuadas por Carraro (1995) por meio de armadilhas iscadas com sardinha, foi inexpressiva. Este autor questionou os comentários que mencionam a inespecificidade dietética do califorídeo, ao refletir sobre a reduzida plasticidade térmica das populações monitoradas por ele e por Ribeiro et al. (1993), os quais sugeriram investigações mais detalhadas sobre a preferência alimentar da espécie.

O presente estudo avaliou, em condições controladas, o desempenho dos imaturos de C. putoria, no que tange à taxa de sobrevivência dos estágios larval e pupal, à razão sexual e à duração do período de desenvolvimento pósembrionário. Para tal, foi disponibilizada, durante a fase ativa de desenvolvimento, dieta de carne equina inata proveniente de animal eutanasiado no Hospital Veterinário da UFRRJ por motivo de falência orgânica. 


\section{MATERIAL E MÉTODOS}

Matrizes coletadas em aviários possibilitaram a formação do estoque fundador da população desse díptero. As neolarvas de $C$. putoria, pertencentes à segunda geração $(\mathrm{F} 2)$, foram transferidas para uma dieta à base de carne equina (tecido muscular). O substrato, previamente armazenado em freezer, foi descongelado em refrigerador, durante 24 horas e, em seguida, alocado em estufa regulada a $30^{\circ} \mathrm{C}$, durante duas horas, objetivando seu pré-aquecimento. $\mathrm{O}$ experimento constou de quatro repetições compostas por 50 neolarvas/repetição, inoculadas em $100 \mathrm{~g}$ de dieta.

Os materiais empregados e os procedimentos metodológicos de rotina seguiram a orientação descrita por Milward-de-Azevedo et al. (1995). Imediatamente após o abandono espontâneo da dieta, as larvas foram individualizadas, o que permitiu o cálculo médio da massa corporal. Essa variável foi registrada 24 horas após a pupariação dos espécimes. Os adultos foram separados por sexo. $\mathrm{O}$ experimento foi monitorado em câmara climatizada regulada a $30^{\circ} \mathrm{C}, 60 \pm 10 \%$ de UR e 14 horas de fotofase. Os registros individualizados de massa corporal dos imaturos (larvas pós-alimentadas e pupas com 24 horas de idade) foram emparelhados e contrastados pelo teste 't'. Admitiram-se, para esses cálculos, 151 pares.

\section{RESULTADOS E DISCUSSÃO}

A duração e a taxa de sobrevivência dos estágios larval e pupal de $C$. putoria foram de cerca de quatro dias e $80 \%$, respectivamente (Tab. 1). A razão sexual foi próxima à esperada: 0,57. Submetida à alternância de temperatura $\left(23^{\circ}\right.$ $29^{\circ} \mathrm{C}$ ), C. putoria desenvolveu-se de ovo a adulto em cerca de 9,5 dias (Greenberg e Skyska, 1984).

Tabela 1. Desenvolvimento pós-embrionário de Chrysomya putoria criada em dieta de carne equina, sob condições controladas $\left(30^{\circ} \mathrm{C}, 60 \pm 10 \%\right.$ UR e 14 horas de fotofase)

\begin{tabular}{|c|c|c|c|c|c|c|}
\hline \multirow{2}{*}{$\begin{array}{c}\text { Características } \\
\text { biológicas }\end{array}$} & \multicolumn{3}{|c|}{$\begin{array}{c}\text { Duração do } \\
\text { desenvolvimento (dias) }\end{array}$} & \multicolumn{2}{|c|}{$\begin{array}{c}\text { Massa corporal }^{1} \\
(\mathrm{mg})\end{array}$} & \multirow{2}{*}{$\begin{array}{c}\text { Sobrevivência } \\
(\%)\end{array}$} \\
\hline & $\overline{\boldsymbol{x}} \pm \sigma \overline{\mathrm{x}}$ & Amplitude & $\begin{array}{l}\text { C.V. } \\
(\%)\end{array}$ & $\overline{\boldsymbol{x}} \pm \sigma \overline{\mathrm{x}}$ & $\begin{array}{l}\text { Intervalo de } \\
\text { variação }\end{array}$ & \\
\hline Estágio larval & $4,16 \pm 0,18$ & $4-5$ & 8,9 & $58,06 \pm 0,44$ & $34,80-72,60$ & 79 \\
\hline Estágio pupal & $4,12 \pm 0,16$ & $4-5$ & 7,8 & $40,10 \pm 0,43$ & $18,70-50,70$ & 81 \\
\hline $\begin{array}{l}\text { Período de } \\
\text { neolarvas ao } \\
\text { abandono } \\
\text { espontâneo dos } \\
\text { espécimes da dieta }\end{array}$ & $3,12 \pm 0,16$ & $3-4$ & 10,6 & & & \\
\hline $\begin{array}{l}\text { Período do } \\
\text { abandono } \\
\text { espontâneo dos } \\
\text { espécimes da dieta } \\
\text { à emergência }\end{array}$ & $5,20 \pm 0,21$ & $5-7$ & 8,1 & & & \\
\hline $\begin{array}{l}\text { Período de } \\
\text { neolarvas à } \\
\text { emergência }\end{array}$ & $8,26 \pm 0,23$ & $8-10$ & 5,6 & & & 64 \\
\hline
\end{tabular}

Contrastada à Chrysomya albiceps (Wiedemann) e à Cochliomyia macellaria (Fabricius), $C$. putoria mostrou-se mais precoce, de acordo com esses autores. Quando monitorou larvas e pupas desse muscoide, criadas, durante a fase ativa, em carne bovina, utilizando a relação inicial de 50 larvas: 50g de dieta, D’Almeida (1994) registrou que o estágio larval ocorreu, em média, em 3,51 dias, e o estágio pupal em 8,51 dias. No entanto, este autor manteve os imaturos a $27 \pm 2^{\circ} \mathrm{C}$ de temperatura e $80 \pm 10 \%$ UR. Destacou que, entre os espécimes amostrados, 90,6\% pupariaram e $82 \%$ 
emergiram, índices de sobrevivência superiores aos obtidos no presente bioensaio.

O manuseio mecânico dos imaturos, durante os procedimentos de rotina, possivelmente contribuiu para o incremento da taxa de mortalidade dos espécimes. As larvas de primeiro (L1) e segundo estádio (L2) apresentaram expressivo efeito de grupo. O comportamento gregário não foi observado durante o terceiro estádio (L3); nesta fase, as larvas apresentaram-se dispersas no meio alimentar.

As larvas pós-alimentadas e as pupas com 24 horas de idade incorporaram, em média, 58,06 e 40,10mg, respectivamente. A correlação entre essas variáveis foi significativa no nível de 0,01 ( $\mathrm{r}=0,6447 ; \mathrm{P}<0,000 ;$ intervalo de confiança: 0,5406 - 0,7293). Com $95 \%$ de confiabilidade, estima-se que, sob as condições de manejo empregadas, a diferença entre os parâmetros médios oscile entre 17,23 e 18,69mg; em média, $17,96 \pm 0,37 \mathrm{mg}$. Os valores obtidos não tenderam à linearidade e caracterizaram mais uma curva do que uma linha (Fig. 1). A relação entre a massa corporal de larvas pós-alimentadas e de pupas com 24 horas de idade e o número relativo de indivíduos encontra-se nas Fig. 2 e 3.

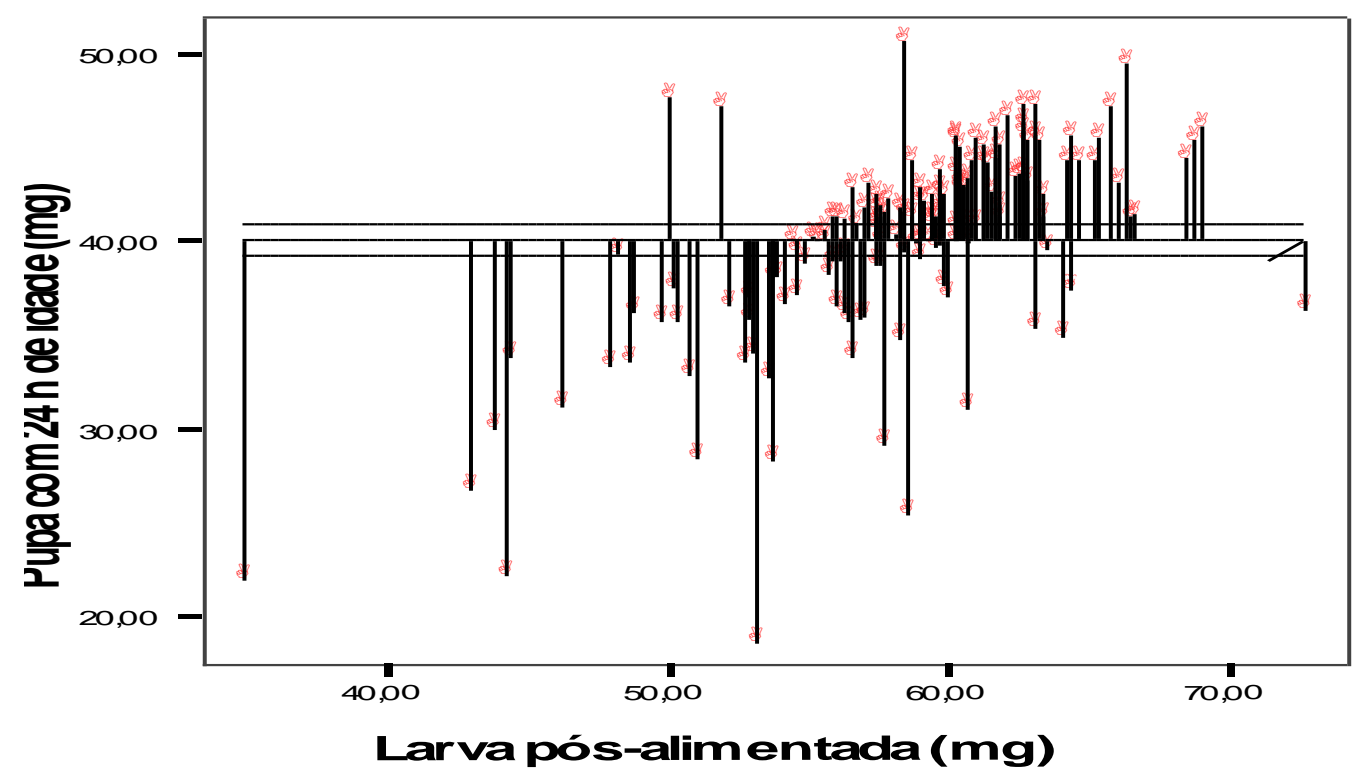

Figura 1. Correlação $(r=0,6447 ; \mathrm{P}<0,0001)$ entre a massa corporal de larvas pós-alimentadas e a massa corporal de pupas com 24 horas de idade de Chrysomya putoria criada em dieta de carne equina, sob condições controladas $\left(30^{\circ} \mathrm{C}, 60 \pm 10 \%\right.$ UR e 14 horas de fotofase). As linhas indicam a média e os intervalos de confiança no nível de $95 \%$.

Os resultados destacados pelo teste ' $t$ ' pareado e o índice de correlação significativa adicionam uma agilidade metodológica ao estudo da espécie-alvo. As larvas pós-alimentadas - e, portanto, caracterizadas pela idade fisiológica - e/ou as pupas com 24 horas de idade - e, portanto, fenologicamente reconhecidas - de C. putoria poderão alternativamente alicerçar, por meio do reconhecimento de sua massa corporal, importantes interpretações sobre o seu desempenho dietético, permitindo contrastes entre diferentes bioensaios consecutivos. A preocupação com o manuseio dos espécimesalvo, durante sua fase ativa de desenvolvimento, deletério ao fator de agregação natural das larvas de califorídeos, oportunamente detalhado por Ullyett (1950), será minimizada, se os objetivos dos bioensaios puderem assimilar os valores obtidos a partir de pré-imagos, de idade conhecida, protegidos pelos pupários. 


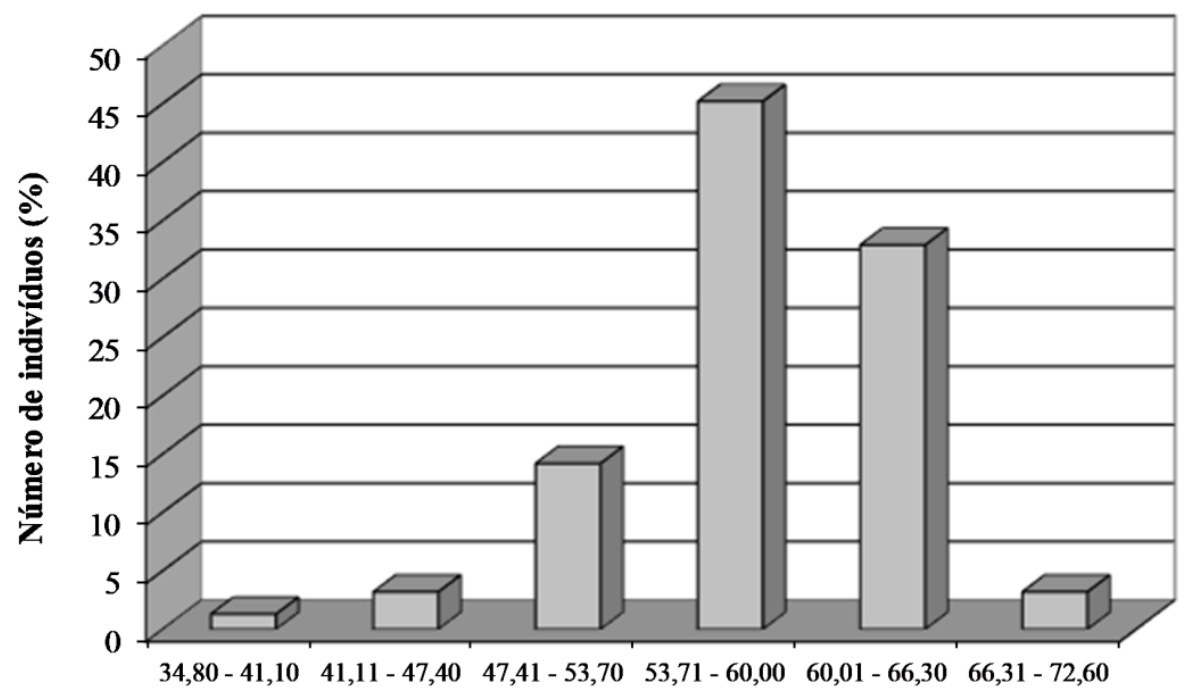

Mass a corporal de larvas (mg)

Figura 2. Relação entre a massa corporal de larvas pós-alimentadas e o número (\%) de imaturos de Chrysomya putoria criados em dieta de carne equina, sob condições controladas $\left(30^{\circ} \mathrm{C}\right.$ de temperatura; 60 $\pm 10 \%$ UR e 14 horas de fotofase).

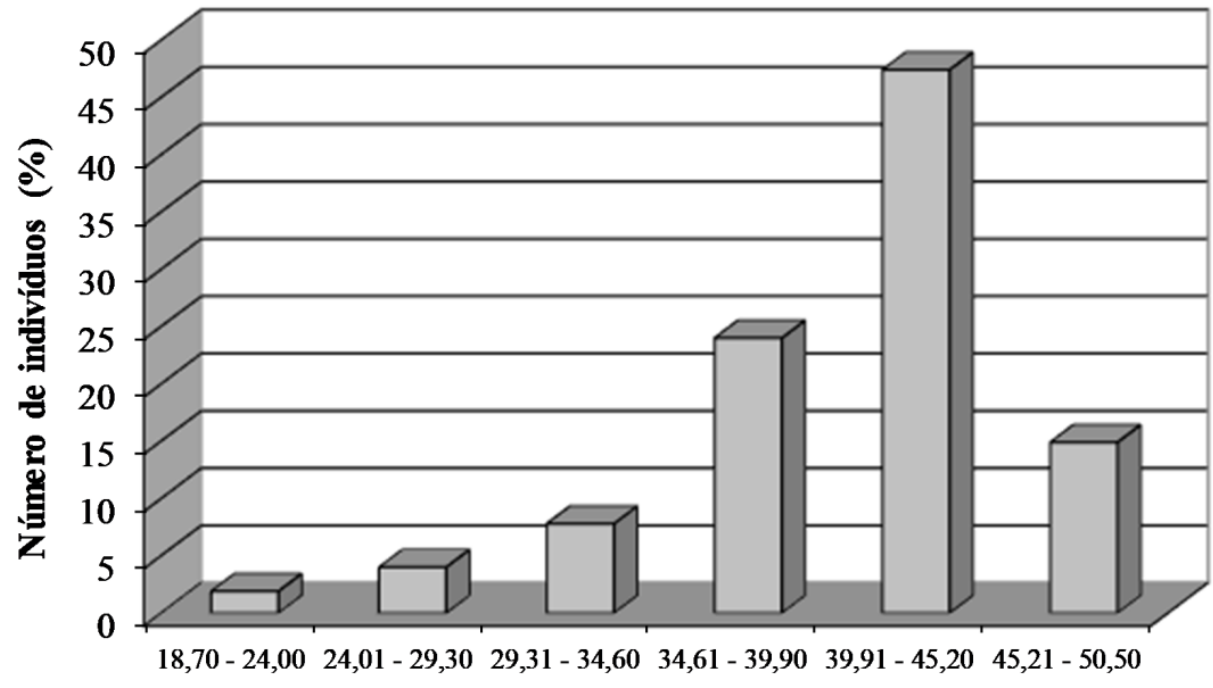

Massa corporal de pupas com 24 horas (mg)

Figura 3. Relação entre a massa corporal de pupas com 24 horas de idade e o número (\%) de imaturos de Chrysomya putoria criados em dieta de carne equina, sob condições controladas $\left(30^{\circ} \mathrm{C}, 60 \pm 10 \%\right.$ UR e 14 horas de fotofase).

\section{CONCLUSÕES}

A carne equina mostrou-se eficiente como dieta para estudos de biologia de califorídeos; da mesma forma, eventuais litígios ou investigações que tratem diretamente da estimativa intervalo pós-morte em cavalos podem ser beneficiados com os dados aqui apresentados. 


\section{REFERÊNCIAS}

BALTAZAR, F.N.; CAVALLARI, M.L.; CARVALHO, E. et al. Entomologia forense e saúde pública: relevância e aplicabilidade. Bepa, v.8, p.14-25, 2011.

CABLK, E.M.; SZELAGOWLSK, E.E.; SAGEBIEL, C.J. Characterization of the volatile organic compounds present in the headspace of decomposing animal remains, and compared with human remains. Forensic Sci. Int., v.220, p.118125, 2012.

CARRARO, V.M. Descrição quantitativa de Chrysomya megacephala, Chrysomya albiceps $e$ $C^{o}$ Chliomyia macellaria (Diptera: Calliphoridae), no Campus da Universidade Federal Rural do Rio de Janeiro, em função da utilização da isca à base de sardinha. 1995. 111f. Dissertação (Mestrado em Medicina Veterinária) - Departamento de Parasitologia Animal, Universidade Federal Rural do Rio de Janeiro, Seropédica, RJ.

CARVALHO, C.J.B.; COURI, M.S. Muscidae, Fanniidae e Calliphoridae (Diptera) do projeto Maracá, Roraima, Brasil. Acta Amazonica, v.21, p.35-43, 1991.

CUNHA, C.L.; LOMÔNACO, C. Monitorização de impacto ambiental provocado por dispersão de moscas em bairros adjacentes a uma granja avícola. An. Soc. Entomol. Bras., v.25, p.1-12, 1996.

D’ALMEIDA, J.M. Substratos de criação de dípteros caliptratos (Calliphoridae, Fanniidae, Muscidae e Sarcophagidae) em condições naturais e de laboratório. 1994. 70f. Tese (Doutorado em Biologia Parasitária) - Instituto Oswaldo Cruz, Fio Cruz, Rio de Janeiro, RJ.

D’ALMEIDA, J.M.; FRAGA, M.B. Efeito de diferentes iscas na atração de califorídeos (Diptera) no campus do Valonguinho, Universidade Federal Fluminense, Niterói, RJ, Brasil. Rev. Bras. Parasitol. Vet., v.16, p.199-204, 2007.

FERRAZ, A.C.P.; DALLAVECCHIA, D.L.; SILVA, D.C. et al. Alternative diets for chrysomya putoria, an old world screwworm fly. J. Insect Sci., v. 12, p.1-11, 2012.

FERREIRA, M.J.M. Sinantropia de Calliphoridae (Diptera) em Goiânia, Goiás. Rev. Bras. Biol., v.43, p.199-210, 1983.
FERREIRA, M.J.M. Sinantropia de dípteros muscóideos de Curitiba, Paraná, I. Calliphoridae. Rev. Bras. Biol., v.38, p.445-454, 1978.

FURLANETTO, S.M.P.; CAMPOS, M.L.C.; HÁRSI, C.M. et al. Microorganismos enteropatogênicos em moscas africanas pertencentes ao gênero Chrysomya (Diptera: Calliphoridae) no Brasil. Rev. Microbiol., v.15, p.170-174, 1984.

GREENBERG, B. Flies and disease: biology and disease transmission. Princeton: Princeton University Press, 1973. v.1, 447p.

GREENBERG, B. Flies and disease: ecology, classification and biotic associations. Princeton: Princeton University Press, 1971. v.1, 865p.

GREENBERG, B.; SZYSKA, M.L. Immature stages and biology of fifteen species of Peruvian Calliphoridae (Diptera). Ann. Ent. Soc. Am., v.77, p.488-517, 1984.

GUIMARÃES, J.H.; PAPAVERO, N. Myiasis in man and animals in the Neotropical Region: bibliographic database. São Paulo: Plêiade, 1999. 308p.

HALL, D.G. The blowflies of North America. Baltimore: The Thomas Say Foundation, 1948. $477 \mathrm{p}$.

HANSKI, I. Nutritional ecology of insects, mites, spiders, and related invertebrates. New York: Wiley, 1987. 1016p.

HULLEY, P.E. A survey of the flies breeding in poultry manure, and their potential natural enemies. J. Entomol. Soc. S. Afr., v.46, p.37-47, 1983.

IMBIRIBA, A.S.; IZUTANI, D.T.; MILHORETTO, I.T.; LUZ, E. Introdução de Chrysomya chloropyga (Wied, 1819) na região Neotropical. Arq. Biol. Technol., v.20, p.35-39, 1977.

LOCKWOOD, J.A.; KUMAR, R.; ECKLES, D.G. Mystery of the slaughtered horse: solving a 400-year-old death with forensic entomology. Am. Entomol., v.40, p.210-215, 1994.

LOMÔNACO, C.; PRADO, A.P. Dispersão de Musca domestica L. e Chrysomya putoria (Wiedemann) em granjas de galinhas poedeiras. An. Soc. Entomol. Bras, v.23, p.179-187, 1994. 
MADEIRA, N.G.; DIAS, E.S.; MASCARENHAS, C.S. Contribuição ao conhecimento da fauna de Calliphoridae (Diptera) Sinantrópicos de Pampulha, Belo Horizonte, Minas Gerais. Rev. Bras. Entomol., v.26, p.137140, 1982.

McGARRY, J.; RATSEP, E.; RESSEL, L. et al. Introducing forensic entomology in cases of suspect animal neglect. Vet. Rec., v.182, p.139, 2017.

MILWARD-DE-AZEVEDO, E.M.V.; HERZOG, J.D.; FREITAS, M.A.S.; FARIA, E.H. Desenvolvimento ontogenético, potencial reprodutivo e longevidade de Chrysomya megacephala (Fabricius) (Diptera, Calliphoridae), em condições de laboratório. Rev. Bras. Entomol., v.39, p.623-632, 1995.

OLIVEIRA-COSTA, J. Insetos peritos - a entomologia forense no Brasil. Campinas: Millennium, 2013. 512p.

PRADO, A.P.; GUIMARÃES, J.H. Estado atual de dispersão e distribuição do gênero Chrysomya Robineau-Desvoidy na região neotropical (Diptera, Calliphoridae). Rev. Bras. Entomol., v.26, p.225-231, 1982.
RIBEIRO, O.B.; PRADO, A.P.; GUIMARÃES, J.H. Competição intra-específica em Chrysomya putoria (Wiedemann, 1830) (Diptera: Calliphoridae) em meio artificial. Rev. Bras. Entomol., v.37, p.641-652, 1993.

SERENO, F.T.P.S.; NEVES, D.P. Ocorrência natural de microhimenópteros parasitóides de pupas de moscas em aviário. An. Soc. Entomol. Brasil, v.22, p.527-533, 1993.

THYSSEN, P.J.; GRELLA, M.D. Efeito da escopolamina sobre o desenvolvimento de Chrysomya putoria (Diptera: Calliphoridae) e sua importância para a estimativa do intervalo pósmorte. Rev. Bras. Criminalistica, v.1, p.39-42, 2011.

ULLYETT, G.C. Competition for food and allied phenomena in sheep-blowfly populations. Philos. Trans. R. Soc. B, v.234, p.77-174, 1950.

WILLIAMS, R.W. A study of filth flies in New York City - 1953. J. Econ. Entomol., v.47, p.556$562,1954$.

ZUMPT, F. Myiasis in man and animals in the old world. London: Butterworths, 1965. 267p. 